\title{
Association between Portal Vein Colored Doppler Ultrasound Findings and Severity of Liver Disease in Cirrhotic Patients with Portal Hypertension
}

Wafek I. Ali ${ }^{1}$ MD, Mohamed I. Hammad ${ }^{1}$ MD, Mohamed Z. Abu Amer ${ }^{2}$ MD, and Islam A. Heikal M.B.B.,Ch ${ }^{1 *}$

*Corresponding Author:

Islam A. Heikal

islamheikal88@gmail.com

Received for publication January 22, 2020; Accepted March 12, 2020; Published online March 12, 2020.

Copyright 2020 The Authors published by Al-Azhar University, Faculty of Medicine, Cairo, Egypt. All rights reserved. This an openaccess article distributed under the legal terms, where it is permissible to download and share the work provided it is properly cited. The work cannot be changed in any way or used commercially.

doi: 10.21608/aimj:2020.22659.1095

Radiodiagnosis ${ }^{l}$ and Tropical Medicine ${ }^{2}$ Departments, Faculty of Medicine, Al-Azhar University Cairo, Egypt.

\begin{abstract}
Background: Chronic liver disease is a heterogeneous and dynamic condition. So, noninvasive estimations of the condition and changes in portal hypertension and hepatic fibrosis are important in the management of this disease.

Aim of the Work: to correlate between color Doppler ultrasound findings of portal vein and severity of liver disease in cirrhotic patients with portal hypertension in the prediction of the future clinical outcomes and prognosis.

Patient and Methods: This study was conducted on 50 patients attending to Al-Hussein University hospital and Damanhur Fever hospital during the time of the study who were divided into groups: Control and Child (A, B, and C).

Results: The study showed an increase in the mean PV diam. with $\mathrm{P}$ value (0.02), marked decrease in PVV with P value $(0.01)$. and increase PV congestive index with p-value (0.002). From child A to child B through child C compared with control and the difference was statistically significant.

Conclusion: Doppler ultrasound has a high diagnostic value of cirrhosis Alterations of liver hemodynamics resulting from liver cirrhosis at the tissue level are detectable on Doppler sonography. Doppler findings of direction flow and velocity of the main PV and congestive index correlate with clinical parameters using the Child Pugh score.

Keywords: Portal Vein; Colored Doppler Ultrasound; Child-Pugh;

Cirrhotic Patients; Portal Hypertension
\end{abstract}

Disclosure: The authors have no financial interest to declare in relation to the content of this article. The Article Processing Charge was paid for by the authors.

Authorship: All authors have a substantial contribution to the article.

\section{INTRODUCTION}

Liver cirrhosis is a progressive liver fibrosis caused by chronic liver disease. It is a prevalent problem in all over the world and leads to significant morbidity and mortality by liver cancer and liver failure. ${ }^{1}$ Early diagnosis can inhibit the associated detrimental signs, including variceal bleeding, hepatic encephalopathy, and portal vein thrombosis. Staging of liver cirrhosis helps with prognostic information and directs appropriate therapy. ${ }^{2}$ Severity of liver disease and cirrhosis can be graded by Child-Pugh (CP) score.
Where the parameters of bilirubin, prothrombin time, serum albumin, presence of ascites and hepatic encephalopathy are accorded individual numerical points (ranging from 1 to 3 ) which are summed up to give the $\mathrm{CP}$ score. This scoring is useful in determining the short-term mortality rate and predicting the waiting list mortality of patients listed for liver transplantation. ${ }^{3}$ Ultrasonography is the worldwide best imaging modality for diagnosis and follows up of cirrhotic patients. The diagnosis usually depends on late findings of liver surface irregularity and secondary findings of portal hypertension. However, B mode sonography is unable to examine patients with cirrhosis without this late findings. ${ }^{4}$ Doppler sonography is a non-invasive modality based on hemodynamic parameters which might have developed even in patients with normal findings on B-mode sonography. ${ }^{5}$ Therefore evaluation of these alterations has a big value for 
early diagnosis and for close follow up of previously diagnosed cases. ${ }^{6}$

This study aimed to correlate between color Doppler ultrasound findings of PV and severity of liver disease in cirrhotic patients with PHT in the prediction of the future clinical outcomes and prognosis.

\section{PATIENT AND METHODS}

This study was conducted between January and September 2019, on 50 patients attending to AlHussein University hospital and Damanhur Fever hospital during the time of the study. The study included patients with hepatitis and cirrhosis with portal hypertension diagnosis was based on a combination of the following: clinical data such as ascites, jaundice, muscle wasting, palmar erythema, cutaneous spider angiomas, flapping tremors, and ecchymosis. Laboratory data including liver synthetic functions such as decreased serum albumin, prolonged prothrombin time and INR, investigations that determine the etiology of the chronic liver disease ( $\mathrm{HBs} \mathrm{Ag}, \mathrm{HBc} \mathrm{Ab}, \mathrm{HCV} \mathrm{Ab}$ ), US findings such as irregular liver surface and coarsened echotexture, splenomegaly, as well as presence of collaterals, portal vein thrombosis and portal cavernomas and patient with HCC. Endoscopy and liver biopsy were not performed in all patients. Noncirrhotic portal hypertension, pregnant women with cirrhosis, patients with grade III and IV encephalopathy, previous endoscopic treatment of varices (sclerotherapy or endoscopic band ligation) and patients who were subjected to previous surgical Porto-systemic shunts or TIPS were excluded from the study.

The work included 2 Groups: Control Group (I) (10 patients), Study Group (II) (40 patients) which subdivided according to the Child-Pugh criteria: Child (A) Subgroup: included 21 patients who were presented by common diffuse forms of liver disease, Child (B) Subgroup included 13 patients with cirrhotic liver and Child (C) Subgroup included 6 cirrhotic patients.

Patients included in the study were subjected to the following: Full medical history with focusing on risk factors especially for viral hepatitis as blood transfusion, dental procedures. Also, the history of symptoms of CLD was checked for. Laboratory investigations: Blood sampling was performed for measuring serum bilirubin, albumin, International Normalized Ratio (INR). Clinical judgments were performed to assess hepatic encephalopathy. Investigations to determine the etiology of chronic liver disease(HBs $\mathrm{Ag}, \mathrm{HBc} \mathrm{Ab}, \mathrm{HCV} \mathrm{Ab}$ ). Abdominal Ultrasonography: All patients fasted for 6 hours before the examination. All measurements were obtained during a short time breath-holding avoiding deep respiration. And all the following were obtained:- Liver size and echo-pattern, Portal vein diameter, Presence of PVT or Portal cavernoma, Spleen span and splenic vein diameter, Presence of ascites, Presence of collateral, GB wall thickness. Assessment patency of PV and blood flow: PV anatomy is evaluated using B-mode imaging which identified by the splenic vein to the right until its junction with the SMV. However, when the PV is difficult to see in the supine position, the patient is examined in the left lateral position. Portal vein diameter and cross-sectional area: Perpendicular to the long axis of the vein, the cross-sectional area was calculated by $(\mathrm{A} \times \mathrm{B}) / 4 \mathrm{x} \Pi$, where $\mathrm{A}$ is the long axis of the vein, $B$ is the short axis of the vein and (П) $=3.14$. IF they were equal, the cross-sectional area was calculated by $\left(r^{2} \times \Pi\right)$, where $r$ is half of the vein diameter. Portal vein flow velocity: The position of the scanner was optimized until a Doppler angle of less than 60 degrees was achieved. The velocity of the PV was calculated from the Doppler tracings. The normal velocity of the PV blood flow was 15-30 $\mathrm{cm} / \mathrm{sec}$. Portal hypertension was associated with an increase in blood flow and congestion, but with a decrease in blood velocity in the PV. Doppler ultrasound was used to show blood flow within PV and its main tributaries and abnormal collaterals. Congestion index: The congestion index (CI) is the ratio between the cross-sectional area $\left(\mathrm{cm}^{2}\right)$ and the blood flow velocity $(\mathrm{cm} / \mathrm{s})$ of the PV. Doppler data were obtained while scanning the PV along its axis and with the sample volume in the mid-portal vein trunk. Just after the Doppler signals were recorded, the PV cross-sectional area was measured from the B-mode image while scanning perpendicular to the long axis of the PV. Image analysis Normal PV diameter is less than $13 \mathrm{~mm}$, with a greater than 20 $30 \%$ increase with food and respiration. In portal hypertension, the PV is dilated $(>13 \mathrm{~mm})$, with absent or less than $20 \%$ variation with respiration. The direction of blood flow can be determined by the color flow pattern. Normal blood flow is toward the liver and is of low velocity, undulating with respiration.

SPSS statistical software package (V. 20, Echosoft Corp., USA) was used for data analysis. Data were expressed as Mean \pm SD for quantitative measures and both number and percentage for categorized data. The following tests were done: Comparison between two independent mean groups for parametric data using Student T-test. Ranked Sperman correlation test to study the possible association between each two variables among each group for non-parametric data. Chi-square test to study the association between every 2 variables or comparison between 2 independent groups as regards the categorized data. The probability of error at 0.05 was considered significant, while at 0.01 and 0.001 are highly significant. Multi Regression analysis was used to 
search for a panel (independent parameters) that can predict the target parameter (dependent variable). By using stepwise multi regression analysis, parameters among these panels can sorted according to their sensitivity to discriminate.

\section{RESULTS}

Analysis of the results of Child's A,B and C patients included in the study showed an increase in the PV mean diam. with $P$ value (0.02), marked decrease in PVV with $P$ value (0.01), and increase in portal vein CI with p-value (0.002), marked increased in spleen span with $\mathrm{P}$ value $(0.003)$ as well as splenic vein diam. With $\mathrm{P}$ value $(0.003)$ from child A to child B through child C compared with control. And there was a statistically significant difference. (Table 1) In addition to PVV in the presence of varices $=13.5 \mathrm{~cm} / \mathrm{sec}$ (16 patients) and PVV in the absence of varices $=14.04 \mathrm{~cm} / \mathrm{sec}$ (24 patients) which showed statistically significant between portal vein velocity in the presence and absence of varices $\mathrm{P}$ value $=0.002$. (Table 2)

Also results of PVV in the absence of ascites $=18.2 \mathrm{~cm} / \mathrm{sec}$ ( 29 patients), PVV in the presence of mild ascites $=14.31 \mathrm{~cm} / \mathrm{sec}$ (6 patients) and PVV in the presence of moderate ascites $=11.06 \mathrm{~cm} / \mathrm{sec}(5$ patients) showed statistically significant between portal vein velocity in the presence and absence of ascites $(\mathrm{p}$ value $=0.001)$. (Table 3$)$

\section{Tables}

\begin{tabular}{|c|c|c|c|c|c|c|}
\hline $\begin{array}{c}\text { VALUE } \\
\text { BY GROUP }\end{array}$ & $\begin{array}{l}\text { Control } \\
(\mathrm{n}=10)\end{array}$ & $\begin{array}{c}\text { Child A } \\
(\mathrm{n}=21)\end{array}$ & $\begin{array}{l}\text { Child B } \\
(\mathrm{n}=13)\end{array}$ & $\begin{array}{c}\text { Child C } \\
(n=6)\end{array}$ & \multicolumn{2}{|c|}{ T-TEST } \\
\hline & $\mathrm{MEAN} \pm \mathrm{SD}$ & $\mathrm{MEAN} \pm \mathrm{SD}$ & $\mathrm{MEAN} \pm \mathrm{SD}$ & $\mathrm{MEAN} \pm \mathrm{SD}$ & $\mathrm{T}$ & P-value \\
\hline P.V diam. & $11 \pm 1.1$ & $13.67 \pm 0.952$ & $15.46 \pm 1.25$ & $15.8 \pm 0.75$ & 2.637 & $0.02 *$ \\
\hline P.V velocity & $24.7 \pm 5.77$ & $19.4 \pm 5.08$ & $14.97 \pm 2.112$ & $11.2 \pm 1.24$ & 2.934 & $0.01 *$ \\
\hline $\begin{array}{l}\text { P.V congestive } \\
\text { index }\end{array}$ & $0.0536 \pm 0.02$ & $0.10 \pm 0.023$ & $0.139 \pm 0.025$ & $0.2 \pm 0.03$ & 4.337 & $0.002^{*}$ \\
\hline Spleen $\operatorname{span}(\mathrm{cm})$ & $10.37 \pm 1.17$ & $12.67 \pm 1.80$ & $14 \pm 0$ & $17 \pm 3.57$ & 14.592 & $0.003^{*}$ \\
\hline $\begin{array}{l}\text { Splenic vein } \\
\text { diam.(mm) }\end{array}$ & $5.2 \pm 1.90$ & $7.23 \pm 1.27$ & $8.96 \pm 1.8$ & $10.066 \pm 0.92$ & 14.592 & $0.003 *$ \\
\hline
\end{tabular}

Table 1: Portal vein diam., PVV, CI of the portal vein, spleen spam and splenic vein diameter in the studied groups.

\begin{tabular}{|c|c|c|c|c|}
\hline \multirow[t]{2}{*}{$\begin{array}{c}\text { VALUE } \\
\text { BY GROUP }\end{array}$} & \multirow{2}{*}{$\begin{array}{c}\text { Presence of varices } \\
\qquad n=16 \\
\text { MEAN }\end{array}$} & \multirow{2}{*}{$\begin{array}{c}\text { absence of varices } \\
\qquad \begin{array}{c}n=24 \\
\text { MEAN }\end{array}\end{array}$} & \multicolumn{2}{|c|}{ T-TEST } \\
\hline & & & $\mathrm{T}$ & P-value \\
\hline P.V. velocity & $13.5 \mathrm{~cm} / \mathrm{sec}$ & $14.04 \mathrm{~cm} / \mathrm{sec}$ & 18.467 & $0.002 *$ \\
\hline
\end{tabular}

Table 2: Relation between portal vein velocity in the presence and absence of varices in the group (II).

\begin{tabular}{|c|c|c|c|c|c|}
\hline \multirow{2}{*}{ VALUE GROUP } & \multicolumn{2}{|c|}{ Presence of ascites } & absence of ascites & \multicolumn{2}{|c|}{ T-TEST } \\
\cline { 2 - 3 } & Mild & Moderate & n=29 \\
& $\mathrm{n}=6$ & $\mathrm{n}=5$ & MEAN \\
& MEAN & MEAN & & \multicolumn{2}{|c|}{ P-value } \\
\cline { 5 - 6 } & & & & 18.034 & $0.001^{*}$ \\
\hline
\end{tabular}

Table 3: Relation between portal vein velocity in the presence and absence of ascites in the group (II). 


\section{DISCUSSION}

Liver biopsy and hepatic venous pressure measurement are the best methods for the assessment of hepatic fibrosis and PHT, respectively, and they have diagnostic and prognostic value. But they are invasive and cannot be used repeatedly in clinical practice. ${ }^{7}$

The ideal noninvasive test must be inexpensive, easy to perform, safe and reproducible as well as to give accurate results in real-time. It should be predictive of long term outcomes related to cirrhosis and PHT to allow prognostic stratification. ${ }^{7}$

Among the noninvasive methods for diagnosis of early liver cirrhosis, ultrasonography has become the most common and valuable method because of its low cost, easy performance, and high acceptability. ${ }^{8}$ In our study, As regards, liver echogenicity, coarse and bright liver were found in child A subgroup while the cirrhotic texture was found in Child B \&C subgroups and this was statistically significant. Also, the wall thickness of GB increased as the disease progress. The wall thickness of GB was significantly higher in cirrhotic patients (Child $\mathrm{B}$ and C). This can be explained by Zheng et al. ${ }^{9}$ who stated that the development of liver fibrosis into cirrhosis is a gradient course. There are nonspecific findings on imaging. However, as the progress of liver fibrosis reaches to a certain degree, the liver pathological changes would become obvious. On ultrasonography, the liver parenchyma echo pattern would become coarse and echogenic and the liver surface would become irregular.

There is a close relationship in histogenesis, anatomy, and function between the biliary system and the liver. Liver cirrhosis often associated with biliary disorders. ${ }^{10}$ This was met in our study as the GB wall thickness was found to be thicker in Child C \& B than Child A patients. Splenomegaly is recognized as a secondary sign of cirrhotic liver. ${ }^{(11)}$ In our study, spleen span as well as splenic vein diam. show statistical significance among the study groups $\mathrm{P}=0.003$.

Assessment of prognosis is an important factor in medical decision making. The Child's classification modified by Pugh et al. ${ }^{12}$ has been established as a valuable indicator of prognosis, Study of portal hemodynamics is important as it provides further insight into the pathophysiology of the disease and helps explore new therapeutic alternatives.

Doppler Ultrasound (DU) and Color Doppler (CD) are routinely included in the abdomen examination because they contribute greatly to the immediate differentiation of vascular and nonvascular structures. Furthermore, DU can detect changes in the direction of blood flow, flow velocity, and organ perfusion. ${ }^{8}$

The disadvantage of duplex US is its operator dependence. For exact measurements, accurate localization of the vessel and a suitable angle are important. Other restrictions include obesity, bowel gases, marked ascites and respiratory movements which prohibit an exact
Doppler examination. ${ }^{13}$

Cirrhosis is combined with increased intrahepatic resistance which increased pressure in the PV enhances the opening up of various collateral pathways. These hemodynamic events are responsible for the progressive fall in the portal venous velocity with increasing severity of the portal hypertension. ${ }^{14}$

In our study, all patients with Child's A subgroup (21 patients) had hepatopetal flow pattern and none of them had hepatofugal flow. In the Child's B subgroup also, none of the patients had hepatofugal flow. One patient of child B subgroup had weak flow due to portal vein thrombosis, In the Child's C subgroup, four patients had hepatopetal flow and two patients had a bidirectional flow pattern (Non hepatopetal flow). So overall two patients (5\%) among the total child patients had non hepatopetal flow (bidirectional flow) with not established yet hepatofugal flow.

In agreements with Von Herbay, ${ }^{15}$ and Wachsberg, ${ }^{16}$ et al. mentioned that the propagation of non hepatopetal flow varied between 3\% and 23\% in the literature which they referred the differences to the proportion of patients with advanced disease and to whether the hepatofugal flow was evaluated in the main PV only versus its major tributaries as well.

In our study, we found that Child's score significant increase in patients with bidirectional flow $(13 \pm 1.47)$ compared to patients with hepatopetal flow (6.66 \pm $2.50)(\mathrm{P}<0.01)$ which is similar to Gaiani et al.'s study which reported that Child-Pugh score was significantly higher in patients with non hepatopetal flow. Our study shows that the propagation of non hepatopedal flow was significantly increased in patients with Child's C cirrhosis.

In our study, average peak venous velocities in the main PV were studied in Child's A, B and C patients. The average PVV in 21 patients with Child's A subgroup was $(19.4 \pm 5.08 \mathrm{~cm} / \mathrm{s})$. Average PVV in 13 patients with Child's B subgroup was $(15 \pm 2.1 \mathrm{~cm} / \mathrm{s})$, while the average PVV in 6 patients with Child's $C$ subgroup was $(11.2 \pm 1.24 \mathrm{~cm} / \mathrm{s})$. It was observed that the average PVV in the Child's B subgroup was significantly decreased than that in the Child's A subgroup $(\mathrm{P}<0.01)$. In addition, average PVV in the Child's C Subgroup was significantly decreased than that in the Child's B and A subgroups $(\mathrm{P}<0.01)$. Similar trends have been mentioned in the literature. ${ }^{18}$

Our study could prove the relation between the fall in PVV and the presence of ascites. The average peak PVV among 11 patients with ascites was $(12.68 \pm 2.72 \mathrm{~cm} / \mathrm{s})$, while the average peak PVV among those patients without ascites (29 patients) was $(18.2 \pm 7.07 \mathrm{~cm} / \mathrm{s})$. This was statistically significant $(\mathrm{P}<0.01)$ which was similar to the trend reported by Chawla, ${ }^{18}$ and Shi, ${ }^{19}$ et al.

In our study as regard lab findings among the studied groups, there is an increase in mean serum bilirubin (3.1 \pm 0.54$)$, and INR $(2.17 \pm 0.19)$ in the child (C) in 
comparison with child (B) \& (A) subgroups and the difference was statistically significant. As well as there is also decrease in mean serum albumin $(2.69 \pm 0.29)$ in the child $(\mathrm{C})$ in comparison with child $(\mathrm{B})(3.19 \pm 0.34)$ \& (A) $(3.84 \pm 0.23)$ subgroups as well as control $(4.051 \pm 0.18)$ and the difference was statistically significant $\mathrm{P}$ value $=0.003$.

In our study, Splenic varices were seen in $40 \%$ while $60 \%$ of the patients have no splenic hilar varices. And none of the patients had gall bladder varices. The mean PVV $(13.5 \mathrm{~cm} / \mathrm{s})$ was significantly lower in patients with splenic varices in comparison to those without splenic varices PVV $(14.04 \mathrm{~cm} / \mathrm{s})(\mathrm{P}<0.05)$. This presentation is logical and can be explained fact that splenic varices provide an alternate route for drainage of the portal venous flow, thereby diminishing the hepatic flow and thereby the portal velocity. With progression, this may lead to hepatofugal flow.

The portal vein $\mathrm{CI}$ shows significantly increased as the disease progress from child A subgroup $(0.10 \pm 0.023)$ to child B subgroup $(0.139 \pm 0.025)$ through child C subgroup $(0.2 \pm 0.04)$, this difference was statistically significant $(\mathrm{P}$ value $=0.002)$.

The portal vein $\mathrm{CI}$ shows evidence of higher sensitivity (71\%) in detecting cirrhosis than PVV $(23 \%)$. By using this index, the PVV was significantly reduced, while the cross-sectional area of the PV was increased in cirrhotic patients. $^{20}$

The mean PVD shows significantly increased as the disease progress from child A subgroup (13.67 \pm 0.952 $\mathrm{mm})$ to child B subgroup $(15.46 \pm 1.25 \mathrm{~mm})$ through child $\mathrm{C}$ subgroup $(15.8 \pm 0.75 \mathrm{~mm})$ (P value $=0.02)$. It is noted that mean PVD shows a significant correlation with CTP score in agreements with a study done by Macias et al. ${ }^{21}$

On the other hand, other studies mentioned that no significant difference in PVD between cirrhotic patients and control patients. ${ }^{18}$ or between the compensated and decompensated cirrhosis groups, or among various CTP grades suggesting that PVD does not correlate with the high portal pressure and the severity of cirrhosis. ${ }^{22}$ Measurement of PVV is an easy, noninvasive procedure and quick. Therefore, a finding of a decrease in PVV below the threshold of $15 \mathrm{~cm} / \mathrm{s}$ may be alone or combined with other factors, an important parameter in the assessment of the risk of PVT (portal vein thrombosis) in cirrhotic patients.

In our study one patient had PVT without a tumor represented about $2.5 \%$ Of cases in group II (PVV=12.1 $\mathrm{cm} / \mathrm{sec}$.); In agreements with Zocco et al. ${ }^{23}$ said that patients with a PVV below $15 \mathrm{~cm} / \mathrm{s}$, may benefit from prophylactic anticoagulation to prevent PVT.

One patient in our study had Hepatocellular Carcinoma with extension to the Main PV (Main Portal Vein Tumor Thrombus)

The accurate frequency of PVT is not known but may occur in as many as $30 \%$ of patients with HCC. Other studies mentioned that the incidence of PVT in HCC varies about $20-30 \%$ in small $\mathrm{HCC}(<3 \mathrm{~cm})$, up to 50 $-75 \%$ in $\mathrm{HCC}>5 \mathrm{~cm} .^{24}$

Using multiple regression analysis the PVV, congestive index, splenic span, splenic vein diam., splenic varices as well as portal vein diam. and ascites shows significant correlation with Child's score, thereby highlighting the importance of PVV, as well as the congestive index in predicting the severity of the disease. However, hemodynamics of PHT is quite complex and PVV alone does not always reflect the degree of hepatic damage and hepatic encephalopathy.

\section{CONCLUSION}

Doppler sonography is a noninvasive technique and helps in the assessment of the degree of liver cirrhosis and the stage of fibrosis, and it should be used to follow the progression of CLD in relation to Child classification. Colour Doppler is an excellent modality for delineating the complex hemodynamics of PHT in cirrhotic patients. Doppler findings of direction, PVV, and CI of PV correlate with clinical parameters using Child-Pugh score.

Limitations of our study include that diagnosis of cirrhosis and PHT was based on the combination of clinical, laboratory and US findings. This could lead to excluding patients with early disease and those with atypical findings. In addition, no follow up has been done.

\section{REFERENCES}

1. Anthony PP. The morphology of cirrhosis: Definition, nomenclature, and classification. Bull WHO 1987; 55:521.

2. Haktanir A, Cihan BS, and Celenk C. Value of Doppler sonography in assessing the progression of chronic viral hepatitis and in diagnosis and grading of cirrhosis. J Ultrasound Med 2005 24: 311-321.

3. Wilson RS and Withers CE. The liver.: Rumack CM, Wilson SR, Charboneau JW, editors. Diagnostic ultrasound. Third ed. St Louis, Missouri: Elsevier Mosby; 2005. p.77-145.

4. Barakat M. Portal vein pulsatility and spectral width in patients with portal hypertension: relation to the severity of liver disease. BJR 2002; 75: 417- 421.

5. Khan AN, Macdonald S, Ali M, et al. Portal Hypertension. eMedicine article. Updated 2002 Dec 18 [cited 2006 Jan 25]. Available from http://www.emedicine.com/ radio/topic570.htm.

6. Pomier-Layrargues G. Hepatic venous pressure gradient measurements: is it mandatory in the management of portal hypertension? J Gastroenterol Hepatol. 2004;19:S158-S161. 
7. Moon Young Kim, Woo Kyoung Jeong, and Soon Koo Baik. Invasive and non-invasive diagnosis of cirrhosis and portal hypertension. World J Gastroenterol 2014; 20(15): 4300-4315 from

http://www.wjgnet.com/10079327/full/v20/i15/ 4300.htm.

8. Martinez-Noguera A, Montserrat E, Torrubia S, et al. Doppler in Hepatic Cirrhosis and Chronic Hepatitis. Elsevier Science; 2002; 23: 1-18.

9. Zheng R, Wang Q, Lu M, et al.: Liver fibrosis in chronic viral hepatitis: An ultrasonographic study. World J of Gastroenterology; 2005; 9: 2484-2489.

10. Wang T, Hwang S and Lee E. Gall bladder wall thickening in patients with liver cirrhosis. $J$ Gastroenterol Hepatol; 1997; 12:445-449.

11. Lim K, Casanova R, Boyer T, et al. Autoimmune hepatitis in African Americans: presenting features and response to therapy. $A m J$ Gastroenterol; 2005 96:3390-3394.

12. Pugh RN, Murray-Lyon IM, Dawson JL, et al. Transection of the oesophagus for bleeding oesophageal varices. Br J Surg. 1973;60(8):6469.

13. Mendoza E chiva, Ein Handbuch, and Arrien Verlag. Duplex Ultrasound of Superficial Leg Veins J Vasc Surg 2002;40:1248-1252

14. Kuo CH, Changchien CS, Tai DI, et al. Portal vein velocity by duplex Doppler ultrasound as an indication of the clinical severity of portal hypertension. Cumulated index medicus. 1995;18(3):217-23

15. von Herbay A, Frieling $\mathrm{T}$ and Haussinger D. Color Doppler sonographic evaluation of spontaneous portosystemic shunts and inversion of portal venous flow in patients with cirrhosis. $J$ Clin Ultrasound. 2000;28(7):332-9.

16. Wachsberg RH, Bahramipour P, Sofocleous CT, et al. Hepatofugal flow in the portal venous system: pathophysiology, imaging findings, and diagnostic pitfalls. Radiographics, 2002;22(1):123-40.

17. Gaiani S, Bolondi L, Li Bassi S, et al.: Prevalence of spontaneous hepatofugal portal flow in liver cirrhosis. Clinical and endoscopic correlation in 228 patients. Gastroenterology. 1991;100 (1):160-7.

18. Chawla Y, Santa N, Dhiman RK, et al. Portal hemodynamics by duplex Doppler sonography in different grades of cirrhosis. Dig Dis Sci. 1998;43(2):354-7.

19. Shi BM, Wang XY, Mu QL, et al. Value of portal hemodynamics and hypersplenism in cirrhosis staging. World J Gastroenterol. 2005;11(5):70811.
20. Moriyasu F, Nishida O, Ban N, et al.: "Congestion index" of the portal vein. AJR Am J Roentgenol. 1986;146(4):735-739.

21. Macias Rodriguez MA, Rendon Unceta P, Navas Relinque C, Tejada Cabrera M, Infantes Hernandez JM, et al.: Ultrasonography in patients with chronic liver disease: its usefulness in the diagnosis of cirrhosis. Rev Esp Enferm Dig. 2003;95(4):258-264,251-257.

22. Ong TZ, Tan HJ. Ultrasonography is not reliable in diagnosing liver cirrhosis in clinical practice. Singapore MedJ. 2003;44(6):293-295.

23. Zocco MA, Di Stasio E, De Cristofaro R, Novi M, Ainora ME, Ponziani F, et al. Thrombotic risk factors in patients with liver cirrhosis: correlation with MELD scoring system and portal vein thrombosis development. J Hepatol 2009;51: 682-689.

24. Yeung YP, Lo CM, Liu CL, Wong BC, Fan ST, Wong J.Natural history of untreated nonsurgical hepatocellular carcinoma. Am J Gastroenterol. 2005;100:1995-2004. 